\title{
Long-term survival of a recurrent gallbladder carcinoma patient with lymph node and peritoneal metastases after multidisciplinary treatments: a case report
}

Koichi Tomita, Kiminori Takano*, Motohide Shimazu, Masaaki Okihara, Toru Sano, Naokazu Chiba and Shigeyuki Kawachi

\begin{abstract}
Background: Gallbladder carcinoma with peritoneal metastasis has a poor prognosis, with a median survival time of 4.8 months. We report the survival of a patient with gallbladder carcinoma with peritoneal metastasis for 7.6 months owing to treatment with tumor resection after chemoradiotherapy.

Case presentation: A 69-year-old man was referred to our hospital for gallbladder carcinoma with hepatic invasion. Cholecystectomy was performed along with S4a and S5 hepatectomy and extrahepatic bile duct resection with lymph node dissection. The postoperative pathological diagnosis was moderately differentiated adenocarcinoma, T3, NO, MO, stage IIIA by the International Union Against Cancer TNM classification. Despite treatment with gemcitabine, the common hepatic artery and para-aortic lymph nodes showed metastases after 3 months from surgery. Although a combination of cisplatin, gemcitabine, and radiotherapy reduced the size of the lymph node metastasis, the peritoneal metastasis persisted. The peritoneal metastasis responded to chemoradiotherapy using tegafur-uracil and leucovorin, but it recurred. The metastasis was resected after 3 years and 9 months from the first surgery, and chemotherapy was discontinued. Seven years and 6 months after the initial surgery, the patient exhibited no signs of tumor recurrence or metastasis.
\end{abstract}

Conclusions: Multidisciplinary treatment including resection without residual tumors could achieve complete remission of gallbladder carcinoma with lymph node and peritoneal metastases in the selected patient.

Keywords: Gallbladder carcinoma, Peritoneal metastasis, Long-term survival, Multidisciplinary treatment, Resection, Chemoradiotherapy

\section{Background}

Gallbladder carcinoma (GBC) is a fatal disease with a poor prognosis, owing to the tendency of the tumor to metastasize early to the regional lymph nodes and spread into the liver bed [1]. GBC with peritoneal metastasis has an exceptionally poor prognosis, with a median survival time (MST) of 4.8 months [2].

Prognostic factors for GBC invasion include liver metastasis, perineural invasion [3], lymphatic invasion, and

\footnotetext{
* Correspondence: kiminoriman526@yahoo.co.jp

Department of Digestive and Transplantation Surgery, Tokyo Medical

University Hachioji Medical Center, 1163 Tatemachi, Hachiojishi, Tokyo 193-0998, Japan
}

lymph node metastasis [4]. Factors associated with survival after $\mathrm{GBC}$ resection include lymph node dissection [5] with extrahepatic bile duct resection [6] and resection of the hepatic bed [7] as well as S4a and S5 hepatectomy [8]. According to these reports, there is no doubt that resection without residual tumors and lymph node dissection or hepatic resection are important for achieving complete remission.

However, GBC with distant metastases to the liver, the lymph nodes beyond the hepatoduodenal ligament, and the peritoneum are typically thought to be contraindications for surgery and are treated best by chemotherapy and radiotherapy, although the MST of patients with

\section{Springer \\ Springer}

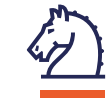

(c) 2016 Tomita et al. Open Access This article is distributed under the terms of the Creative Commons Attribution 4.0

International License (http://creativecommons.org/licenses/by/4.0/), which permits unrestricted use, distribution, and

reproduction in any medium, provided you give appropriate credit to the original author(s) and the source, provide a link to the Creative Commons license, and indicate if changes were made. 
such disease is less than 1 year [2]. We report the survival of a patient with GBC with lymph node and peritoneal metastasis for 7.6 months; this was achieved by controlling disease progression with chemoradiotherapy and resection of the peritoneal metastasis.

\section{Case presentation}

A 69-year-old man was diagnosed with GBC with hepatic invasion after a 2-year follow-up for right breast cancer surgery, during which his serum carcinoembryonic antigen (CEA) level was elevated. He was referred to our hospital in Jun. 2007.

Preoperatively, abdominal ultrasonography and computed tomography (CT) scan revealed a tumor $(37 \times$ $30 \mathrm{~mm}$ in diameter), involving the liver bed from the neck of the gallbladder (Fig. 1). Endoscopic ultrasonography did not reveal any cystic duct invasion; however, metastasis to the lymph node near the common bile duct was suspected on CT scan. Distant metastasis, including to the liver, was not observed. The results of a blood test on admission indicated that liver and kidney functions were normal. The levels of tumor markers were as follows: CEA, $10.3 \mathrm{ng} / \mathrm{mL}$ and carbohydrate antigen (CA) 19-9, 785.24 U/mL.

Cholecystectomy with hepatectomy of S4a and S5, lymph node dissection of the hepatoduodenal ligament, resection of the extrahepatic bile duct, and Roux-en-Y choledochojejunostomy were performed in Jul. 2007. R0 resection was accomplished. The postoperative course was uneventful.

According to the seventh edition of the Tumor Nodes and Metastasis (TNM) Classification of the International Union Against Cancer, the postoperative pathological diagnosis was moderately differentiated adenocarcinoma of the gallbladder, T3, N0, M0, stage IIIA (Fig. 2).

The patient's clinical course and associated changes in tumor markers are illustrated in Fig. 3. Adjuvant chemotherapy was administered (gemcitabine [GEM], $1000 \mathrm{mg} / \mathrm{m}^{2}$, biweekly). After 8 cycles of chemotherapy, the patient's CA 19-9 level had increased to $210.51 \mathrm{U} / \mathrm{mL}$ and metastases to the common hepatic artery lymph nodes and paraaortic lymph nodes were detected in Oct. 2007 (3 months post-surgery) on CT scan. Therefore, the chemotherapy regimen was changed to GEM $\left(1000 \mathrm{mg} / \mathrm{m}^{2}\right.$, days 1 and 8 ) and TS-1 (a combination capsules of tegafur, gimeracil, and oteracil potassium, $60 \mathrm{mg} / \mathrm{m}^{2}$, daily, 2 weeks on/ 1 week off). GEM and TS-1 were discontinued when the patient developed thrombopenia accompanied by elevation of CA 19-9 to $801 \mathrm{U} / \mathrm{mL}$ in Jan. 2008. A CT scan revealed that the common hepatic artery lymph node and para-aortic lymph node metastases had increased in size by surrounding the common and proper hepatic arteries; the metastases reached the portal vein bifurcation (Fig. 4).
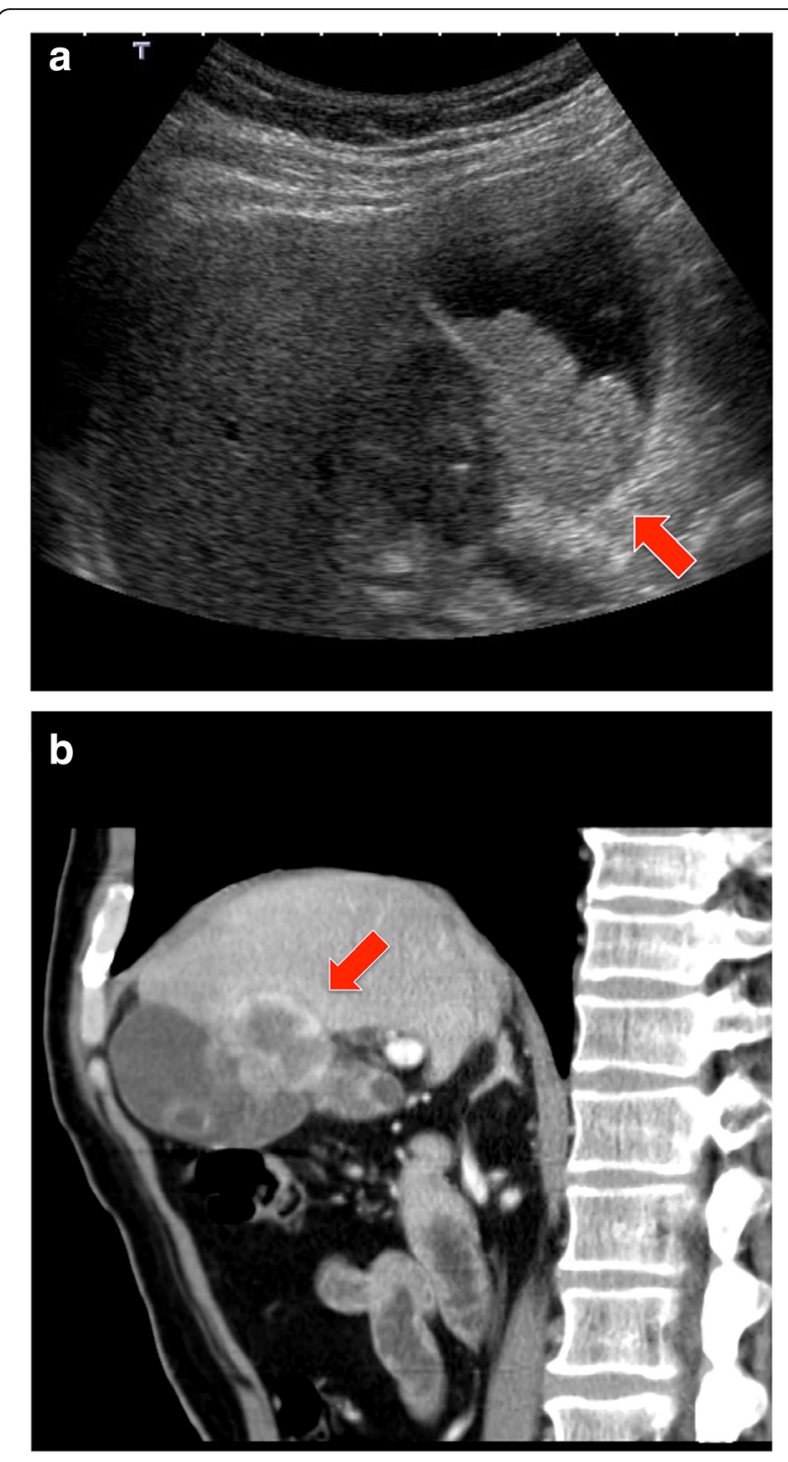

Fig. 1 An ultrasonography image (a) and a sagittal section of a computed tomography scan (b) showing the gallbladder carcinoma. The red arrows indicate the tumor mass at the neck of the gallbladder, $37 \times 30 \mathrm{~mm}$ in diameter, involving the liver bed

In Mar. 2008 (8 months post-surgery), a CT scan revealed an enlargement of the lymph node metastasis with invasion into the portal vein, celiac artery, superior mesenteric artery, duodenum, pancreas, and inferior vena cava. A combination of low-dose cisplatin (CDDP, $6 \mathrm{mg} /$ body, daily) and X-ray radiation consisting of $50 \mathrm{~Gy} / 25 \mathrm{fr}$ was administered. After chemotherapy and radiation were completed in Jun. 2008 (11 months postsurgery), CA 19-9 levels had decreased to $22.53 \mathrm{U} / \mathrm{mL}$.

After the decrease in CA 19-9 levels, GEM $\left(1000 \mathrm{mg} / \mathrm{m}^{2}\right)$ and CDDP $\left(15 \mathrm{mg} / \mathrm{m}^{2}\right)$ were re-administered on days 1 and 8 ( 2 weeks on/1 week off). CA 19-9 decreased to normal levels after three courses of treatment. GEM and CDDP were continued, and stable disease was 


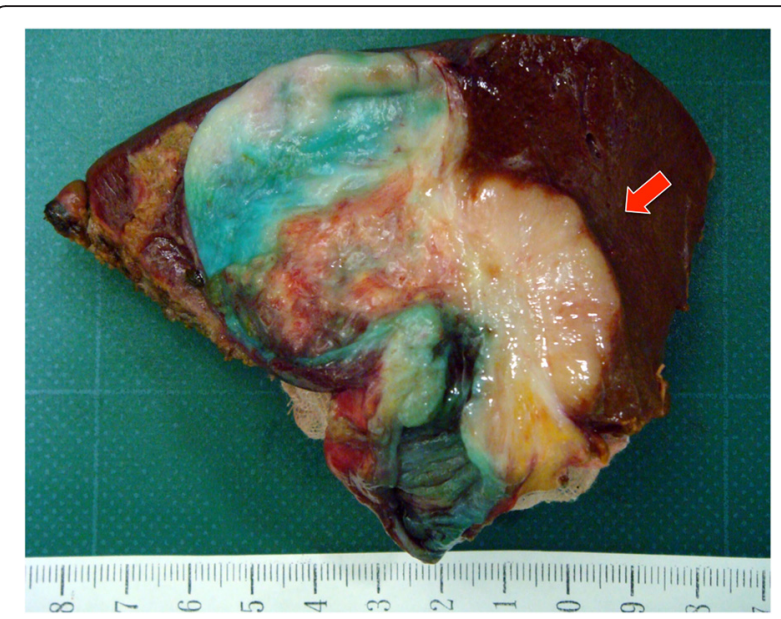

Fig. 2 The cut surface of the resected specimen. The red arrow indicates the tumor mass at the neck of the gallbladder involving the liver bed, which was pathologically diagnosed as a moderately differentiated adenocarcinoma. The lymph node, cystic duct, cystic artery, and other surrounding organs were negative for invasion. Hepatic metastasis was not observed. The gallbladder was injected with indocyanine green, which was used to observe the cystic vein perfusion area

maintained according to the Response Evaluation Criteria in Solid Tumor (RECIST). In Dec. 2009 (2 years and 5 months post-surgery), after 28 courses of treatment had been administered in total, a new metastatic lesion was detected near the colon of the hepatic flexure on a CT scan and was diagnosed as a peritoneal metastasis.

Owing to the discovery of the additional metastasis, the chemotherapy regimen was changed to uracil and tegafur (UFT, $300 \mathrm{mg} /$ body) and leucovorin (25 mg/body), which were administered daily (4 weeks on/1 week off), in Jan. 2010 ( 2 years and 6 months post-surgery). X-ray radiation of $40 \mathrm{~Gy} / 20 \mathrm{fr}$ was also administered. After treatment, the levels of tumor markers decreased to normal, and a CT scan revealed reduced tumor size of the peritoneal metastasis (Fig. 5).

In Apr. 2011 (3 years and 9 months post-surgery), after 16 courses of UFT and leucovorin treatment, the levels of tumor markers had once again increased and the size of the peritoneal metastasis had increased. Radiotherapy treatment was no longer possible because the patient had reached the maximum dose allowable and because of the risk for adverse gastrointestinal effects. Multiple imaging modalities including CT, magnetic resonance imaging (MRI), and positron emission tomography (PET/CT) revealed that the peritoneal metastasis was isolated. Also, the lymph node metastases of hepatoduodenal ligament and para-aorta maintained stable disease and did not show any viability. Therefore, the peritoneal metastasis was resected in Apr. 2011. Open laparotomy findings did not reveal any peritoneal dissemination except the isolated peritoneal metastasis. The metastatic mass was $18 \times 15 \mathrm{~mm}$ in size and was resected without a residual tumor. The postoperative pathological diagnosis was a metastatic adenocarcinoma. The effect of radiotherapy was also observed, and its effect was evaluated as grade $1 \mathrm{~b}$ (about half of tumor cells showed highly change).

After resection, chemotherapy was discontinued because the tumor marker levels were within normal limits. PET/CT scan obtained in Nov. 2011 did not reveal any tumors.

At the time of reporting, 7.6 months have passed since the primary tumor was removed, and 3 years and 9 months have passed since the peritoneal metastasis was resected. At this time, the levels of tumor markers are within normal limits and tumor recurrences have not been detected on CT scan.

\section{Discussion}

In this report, we demonstrate a rare case of long-term disease-free survival in a patient with recurrent $\mathrm{GBC}$ with lymph node and peritoneal metastases by using multidisciplinary treatments including resection, chemotherapy, and radiotherapy.

The poor prognosis of advanced GBC, such as that with distant metastasis to the liver, peritoneum, or lymph nodes of the extrahepatoduodenal ligament or para-aorta, is well recognized. Because of the difficulty associated with complete resection, chemotherapy or radiotherapy is usually utilized in these advanced GBC cases [9]. The main chemotherapeutic agents for GBC include GEM, CDDP, and 5-fluorouracil, alone or in combination. According to a phase II study, $1000 \mathrm{mg} / \mathrm{m}^{2}$ of GEM elicited a $17.5 \%$ response rate (RR) with an MST of 7.6 months in cases of advanced GBC [10]. TS-1 elicited a $35 \%$ RR with an MST of 9.4 months [11]. The combination of GEM and CDDP elicited a 21 to $48 \% \mathrm{RR}$ with an MST of 4.6 to 11.0 months in several reports [12-15]. Although these were cohort studies, randomized trials evaluating the effect of these drugs alone and with other agents, such as epirubicin, etoposide, leucovorin, mitomycin C, and capecitabine, have been performed but have not demonstrated positive outcomes [16-18]. Adjuvant chemotherapy has also been thought to play a marginal role in the treatment of GBC.

Thus, resection without residual tumors, if possible, still has an important role in improving the prognosis of advanced GBC. Several previous reports support the notion that radical resection of advanced GBC enhances survival [19-21]. Watanabe et al. reported the survival of a patient with advanced GBC for more than 12 years after curative surgery that included cholecystectomy, liver bed resection, pancreatoduodenectomy, right hemicolectomy, and anterior abdominal wall resection [22]. 


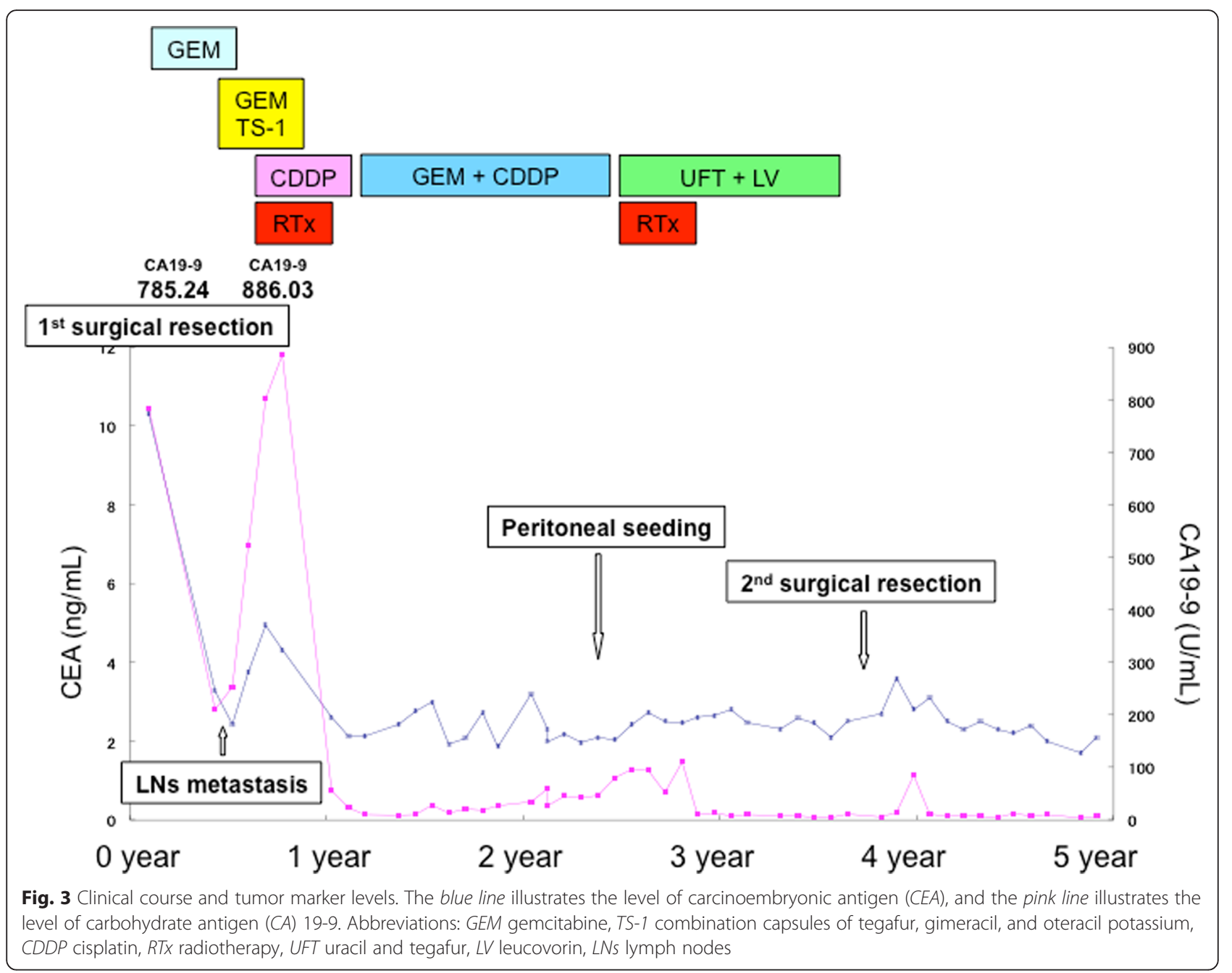

Even in cases of metastatic GBC, if the metastatic lesions were surgically resectable and well controlled with chemotherapy and/or radiotherapy, resection with negative margins might result in long-term survival and complete remission. A previous report showed that patients with advanced GBC could benefit from resection even when para-aortic lymph node metastasis and/or liver metastasis are present [2]. Scaringi et al. reported a patient with recurrent $G B C$ who was treated with iterative resection and was alive and disease-free 5 years after the final surgical procedure [23]. In their case, fluorouracil and leucovorin were administered as adjuvant chemotherapy; however, splenic metastasis appeared after 1 year from the first operation. Even though the splenic metastasis was resected, pancreatic and gastric metastases appeared after another year. Distal pancreatectomy en bloc with sleeve gastrectomy was performed, and the patient was alive for 5 years. Only a few reports have demonstrated the long-term survival of patients with recurrent GBC treated with resection of metastatic lesions, similar to our case.
Our current case demonstrated that long-term survival could be achieved with resection of metastatic lesions of $\mathrm{GBC}$, if the metastatic lesion was isolated, resectable, and well controlled with chemoradiotherapy. The criteria to confirm the isolation of metastasis and the definition of "well controlled" are controversial, and multiple imaging modalities including CT, MRI, and PET/CT could be helpful to confirm it. Also, the definition of resectability is different between surgeons or institutions, and further investigations are needed to provide consistent evidence.

\section{Conclusions}

In conclusion, despite significant developments in the fields of chemotherapy and/or radiotherapy, resection without residual tumor might have an important role in improving the prognosis of recurrent GBC. Resection should be considered if the metastatic lesion is isolated and well controlled with chemoradiotherapy in order to achieve complete remission and/or long-term survival. 


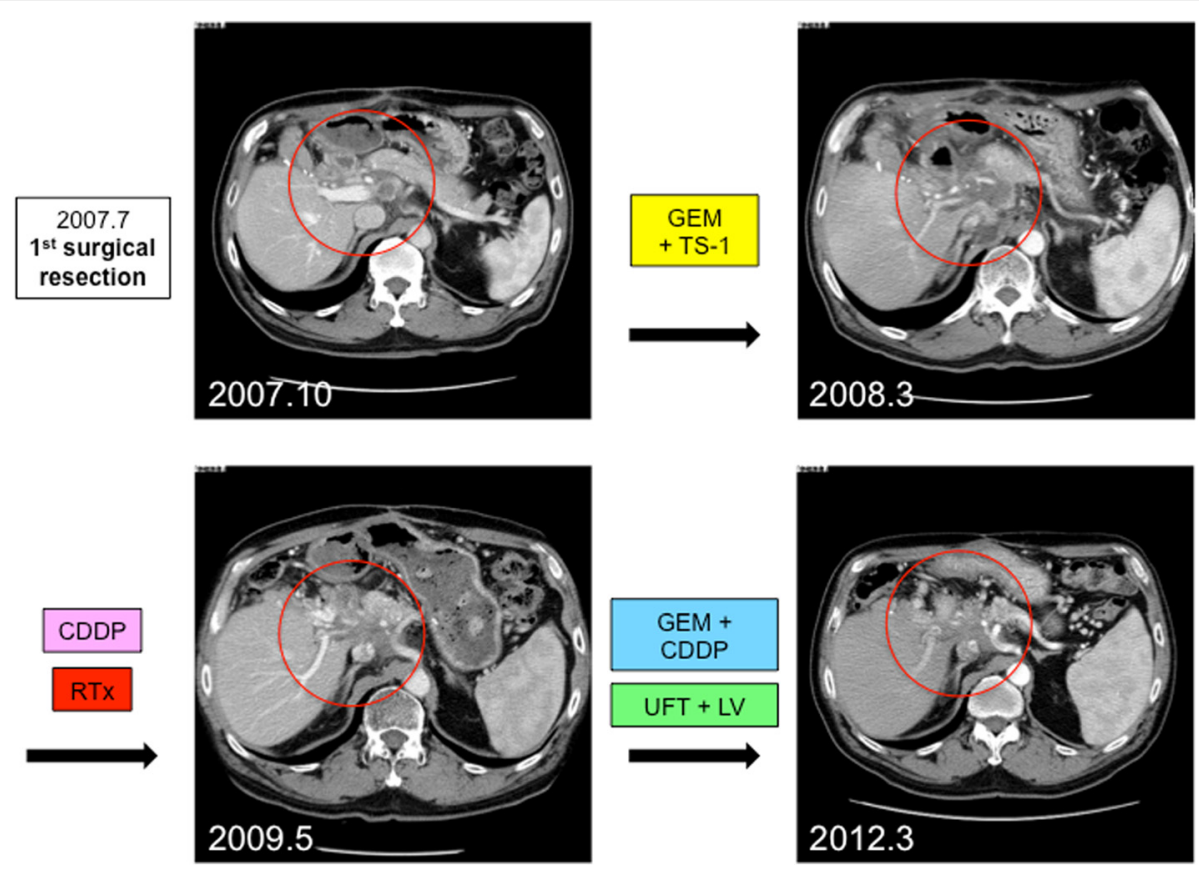

Fig. 4 Progression of the metastasis to the common hepatic artery and para-aortic lymph nodes. The lymph node metastasis was detected 3 months after the first resection (a). Despite administration of gemcitabine (GEM) plus TS-1 and cisplatin (CDDP) plus radiation, the metastasis enlarged and involved the portal vein (b). However, the growth rate remarkably decreased after GEM plus CDDP (c) and uracil and tegafur plus leucovorin (d) administration and has maintained the same size

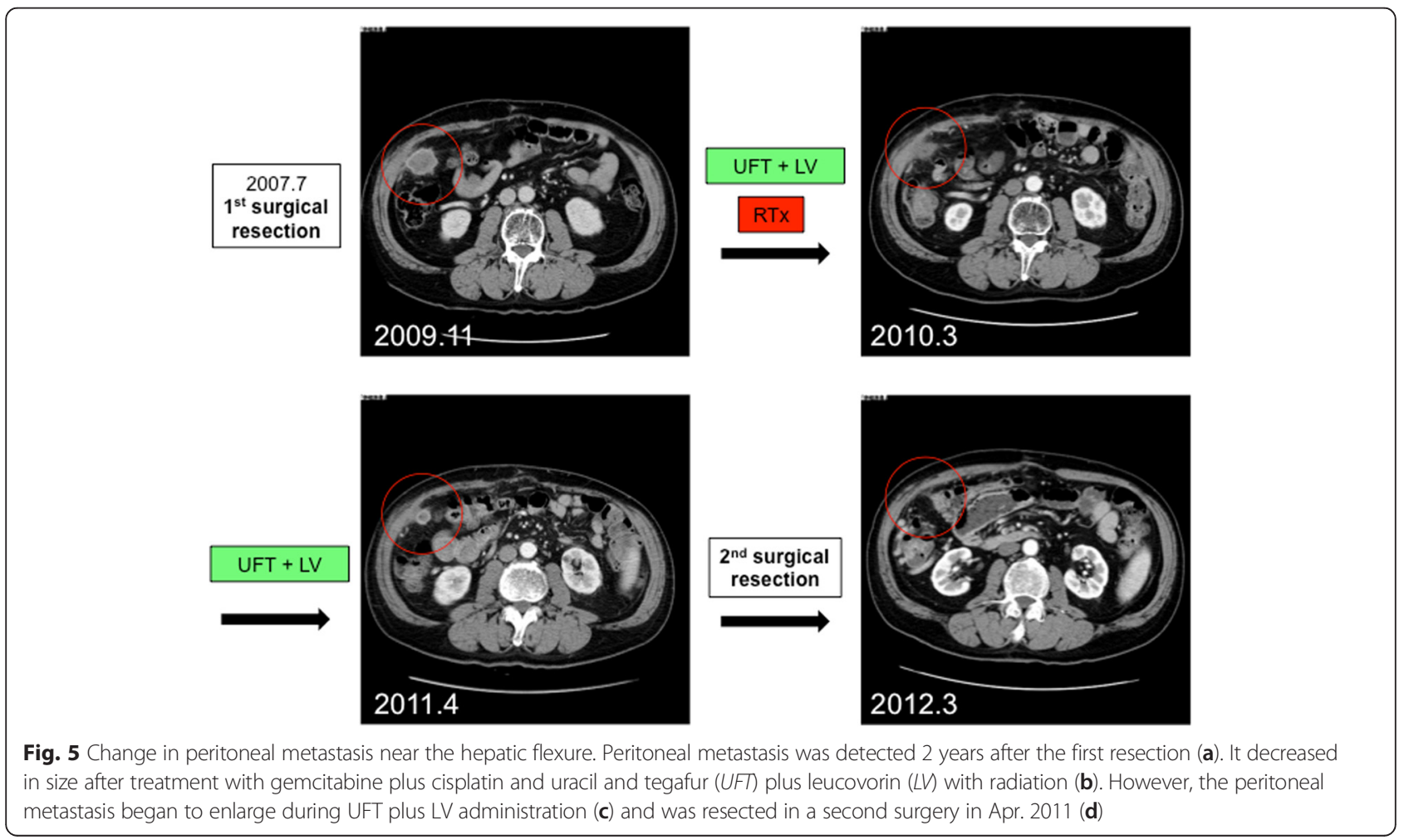




\section{Consent}

Written informed consent was obtained from the patient for publication of this case report and any accompanying images. A copy of the written consent is available for review by the Editor-in-Chief of this journal.

\section{Competing interests}

The authors declare that they have no competing interests.

\section{Authors' contributions}

KTomita and KTakano made the conception and design of this case report. Authors other than KTomita and KTakano (MO, TS, NC, MS, SK) contributed to the collection, analysis, and interpretation of the data. KTomita and KTakano wrote the draft manuscript, and other authors performed the critical revision of the manuscript. All authors gave final approval of the version to be published. KTakano has overall responsibility and guarantees the scientific integrity.

Received: 24 November 2015 Accepted: 1 February 2016 Published online: 11 February 2016

\section{References}

1. Bartlett DL. Gallbladder cancer. Semin Surg Oncol. 2000:19(2):145-55.

2. Nishio $H$, Nagino $M$, Ebata $T$, Yokoyama $Y$, Igami T, Nimura Y. Aggressive surgery for stage IV gallbladder carcinoma; what are the contraindications? J Hepatobiliary Pancreat Surg. 2007;14(4):351-7.

3. Sakamoto Y, Kosuge T, Shimada K, Sano T, Hibi T, Yamamoto J, et al. Clinical significance of extrahepatic bile duct resection for advanced gallbladder cancer. J Surg Oncol. 2006;94(4):298-306.

4. Choi SB, Han HJ, Kim WB, Song TJ, Suh SO, Choi SY. Surgical strategy for T2 and T3 gallbladder cancer: is extrahepatic bile duct resection always necessary? Langenbecks Arch Surg. 2013;398(8):1137-44.

5. Kokudo N, Makuuchi M, Natori T, Sakamoto Y, Yamamoto J, Seki M, et al. Strategies for surgical treatment of gallbladder carcinoma based on information available before resection. Arch Surg. 2003;138(7):741-50. discussion 50

6. Shimizu Y, Ohtsuka M, Ito H, Kimura F, Shimizu H, Togawa A, et al. Should the extrahepatic bile duct be resected for locally advanced gallbladder cancer? Surgery. 2004;136(5):1012-7. discussion 8.

7. Horiguchi A, Miyakawa S, Ishihara S, Miyazaki M, Ohtsuka M, Shimizu H, et al. Gallbladder bed resection or hepatectomy of segments $4 a$ and 5 for pT2 gallbladder carcinoma: analysis of Japanese registration cases by the study group for biliary surgery of the Japanese Society of Hepato-BiliaryPancreatic Surgery. J Hepatobiliary Pancreat Sci. 2013;20(5):518-24.

8. Kohya N, Miyazaki K. Hepatectomy of segment 4a and 5 combined with extra-hepatic bile duct resection for T2 and T3 gallbladder carcinoma. J Surg Oncol. 2008;97(6):498-502

9. Mekeel KL, Hemming AW. Surgical management of gallbladder carcinoma: a review. J Gastrointest Surg. 2007;11(9):1188-93.

10. Okusaka T, Ishii H, Funakoshi A, Yamao K, Ohkawa S, Saito S, et al. Phase II study of single-agent gemcitabine in patients with advanced biliary tract cancer. Cancer Chemother Pharmacol. 2006:57(5):647-53.

11. Ueno H, Okusaka T, Ikeda M, Takezako Y, Morizane C. Phase II study of S-1 in patients with advanced biliary tract cancer. Br J Cancer. 2004;91(10):1769-74.

12. Doval DC, Sekhon JS, Gupta SK, Fuloria J, Shukla VK, Gupta S, et al. A phase II study of gemcitabine and cisplatin in chemotherapy-naive, unresectable gall bladder cancer. Br J Cancer. 2004;90(8):1516-20.

13. Thongprasert S, Napapan S, Charoentum C, Moonprakan S. Phase II study of gemcitabine and cisplatin as first-line chemotherapy in inoperable biliary tract carcinoma. Ann Oncol. 2005;16(2):279-81.

14. Kim ST, Park JO, Lee J, Lee KT, Lee JK, Choi SH, et al. A phase II study of gemcitabine and cisplatin in advanced biliary tract cancer. Cancer. 2006; 106(6):1339-46.

15. Park BK, Kim YJ, Park JY, Bang S, Park SW, Chung JB, et al. Phase II study of gemcitabine and cisplatin in advanced biliary tract cancer. J Gastroenterol Hepatol. 2006;21(6):999-1003.

16. Kornek GV, Schuell B, Laengle F, Gruenberger T, Penz M, Karall K, et al. Mitomycin C in combination with capecitabine or biweekly high-dose gemcitabine in patients with advanced biliary tract cancer: a randomised phase II trial. Ann Oncol. 2004;15(3):478-83.
17. Ducreux M, Van Cutsem E, Van Laethem JL, Gress TM, Jeziorski K, Rougier P, et al. A randomised phase II trial of weekly high-dose 5-fluorouracil with and without folinic acid and cisplatin in patients with advanced biliary tract carcinoma: results of the 40955 EORTC trial. Eur J Cancer. 2005; 41(3):398-403.

18. Rao S, Cunningham D, Hawkins RE, Hill ME, Smith D, Daniel F, et al. Phase III study of 5FU, etoposide and leucovorin (FELV) compared to epirubicin, cisplatin and 5FU (ECF) in previously untreated patients with advanced biliary cancer. Br J Cancer. 2005;92(9):1650-4.

19. Kondo S, Nimura Y, Hayakawa N, Kamiya J, Nagino M, Uesaka K. Extensive surgery for carcinoma of the gallbladder. Br J Surg. 2002;89(2):179-84.

20. Taner CB, Nagorney DM, Donohue JH. Surgical treatment of gallbladder cancer. J Gastrointest Surg. 2004;8(1):83-9. discussion 9.

21. Dixon E, Vollmer Jr CM, Sahajpal A, Cattral M, Grant D, Doig C, et al. An aggressive surgical approach leads to improved survival in patients with gallbladder cancer: a 12-year study at a North American Center. Ann Surg. 2005;241(3):385-94.

22. Watanabe M, Yamazaki K, Yajima S, Tsuchiya M, Otsuka Y, Tamura A, et al. Fourteen-years of disease-free survival in a patient with advanced gallbladder carcinoma after radical resection: a case report. Hepatogastroenterology. 2009;56(90):335-8.

23. Scaringi S, Nesi G, Bargellini T, Batignani G, Tonelli F. Iterative surgical resection of a recurrent gallbladder carcinoma with long-term survival: report of a case. In Vivo. 2010;24(2):215-7.

\section{Submit your manuscript to a SpringerOpen ${ }^{\circ}$ journal and benefit from:}

- Convenient online submission

- Rigorous peer review

- Immediate publication on acceptance

- Open access: articles freely available online

- High visibility within the field

- Retaining the copyright to your article

Submit your next manuscript at $>$ springeropen.com 